\title{
Even-odd effect in Andreev Transport through a Carbon Nanotube Quantum Dot
}

\author{
A. Eichler, M. Weiss, S. Oberholzer, and C. Schönenbergen ${ }^{*}$ \\ Institut für Physik, Universität Basel, Klingelbergstr. 82, CH-4056 Basel, Switzerland \\ A. Levy Yeyati and J. C. Cuevas \\ Departamento de Fisica Teorica de la Materia Condensada, \\ Universidad Autonoma de Madrid, E-28049 Madrid, Spain.
}

(Dated: October 27, 2018)

\begin{abstract}
We have measured the current $(I)$-voltage $(V)$ characteristics of a single-wall carbon nanotube quantum dot coupled to superconducting source and drain contacts in the intermediate coupling regime. Whereas the enhanced differential conductance $d I / d V$ due to the Kondo resonance is observed in the normal state, this feature around zero bias voltage is absent in the superconducting state. Nonetheless, a pronounced even-odd effect appears at finite bias in the $d I / d V$ sub-gap structure caused by Andreev reflection. The first-order Andreev peak appearing around $V=\Delta / e$ is markedly enhanced in gate-voltage regions, in which the charge state of the quantum dot is odd. This enhancement is explained by a 'hidden' Kondo resonance, pinned to one contact only. A comparison with a single-impurity Anderson model, which is solved numerically in a slave-boson meanfield ansatz, yields good agreement with the experiment.

PACS numbers: 74.78.Na,74.45.+c,73.63.Kv,73.21.La,73.23.Hk,73.63.Fg
\end{abstract}

There is a growing interest in the exploration of correlated charge transport through nanoscaled lowdimensional systems involving both superconductors and normal metals $[1,2,3,4,45,6]$. The penetration of the pair amplitude $\Delta$ from a superconductor (S) into a normal metal $(\mathrm{N})$, the proximity effect, is a manifestation of correlated charge transport mediated by Andreev processes taking place at the S-N interface 7] and leading in S-N-S junctions to the Josephson effect [8] and sup-gap current peaks due to multiple Andreev reflection (MAR) [9]. The superconducting proximity effect has been studied in great detail in the mesoscopic size regime of diffusive, but phase coherent conductors [10]. Andreev transport has also been the key quantity in experiments elucidating charge transport in single atom contacts [5, 11]. On the other hand, Andreev transport through a quantum dot coupled to superconductors, is just emerging now [12, 13, 14, 15]. If the dot is weakly coupled to the leads, Andreev processes are suppressed by the charging energy $U$ of the dot [3, 16, 17]. If the dot is sufficiently small, size quantization takes place, forming a quantum dot (QD) with discrete eigenstates ('levels') at energies $E_{\{i\}}$. Transport then occurs through individual levels [3]. Since the level 'positions' $E_{\{i\}}$, and sometimes also the coupling strengths of the levels to both source and drain contacts $\Gamma_{1,2}$, can be tuned through gate voltages, a physically tunable model system of the Anderson 'impurity problem' is realized. With one electron on the QD (half-filling), a many-electron ground-state forms, involving both the dot-state and conduction electrons from the leads in an energy window given by the the Kondo temperature $T_{K}$ [18, 19]. In

*Electronic address: Christian.Schoenenberger@unibas.ch this Kondo regime, which can be observed if $\Gamma_{1,2}$ is not too small, a resonance pinned at the Fermi energy of the leads forms (Kondo resonance). If superconducting contacts are used instead of normal ones, the additional pair-correlation in the leads competes with the Kondo correlations on the QD [1, 2, 20, 21, 22, 23]. It has been found recently in experiments using carbon nanotubes (CNTs) as QDs, that there is an interesting cross-over occurring at $k_{B} T_{K} \approx \Delta$. If $\Delta>k_{B} T_{K}$, the Kondo correlations are suppressed, whereas they persist in the opposite regime, opening a highly conducting channel for the Josephson effect [2, 12]. CNTs are ideally suited for the realization of such systems, because CNTs can a) act as well controlled QDs in different transport regimes [24], including the Kondo regime 25], and b), different types of contacts can be realized, including superconducting ones [4, 6, 12, 13, 14, 15]. Similar physics can be addressed with QDs fabricated in semiconducting nanowires contacted to $\mathrm{S}$ contacts [26].

We here report on measurements of the nonequilibrium (finite-bias) transport through a single-wall carbon nanotube QD with S contacts in the most interesting regime of intermediate coupling, where Kondo correlations are of similar magnitude as superconducting ones. We have found a pronounced even-odd effect in the MAR structure.

Single walled carbon nanotubes were grown by chemical vapor deposition on highly-doped Si wafers with a $400 \mathrm{~nm}$ layer of thermal oxide on top, using Fe particles as catalysts 27, 28]. Individual single-wall carbon nanotubes (SWCNTs) were localized with a scanning electron microscope and contacted to supercondcuting source and a drain electrodes using e-beam lithography, see Fig. 1. The evaporated contacts consist of a $\operatorname{Ti}(5 \mathrm{~nm}) / \mathrm{Al}(100 \mathrm{~nm}) / \operatorname{Ti}(10 \mathrm{~nm})$ tri-layer, where $\mathrm{Ti}$ serves as an adhesion and cap-layer. $\mathrm{Al}$ is the ac- 


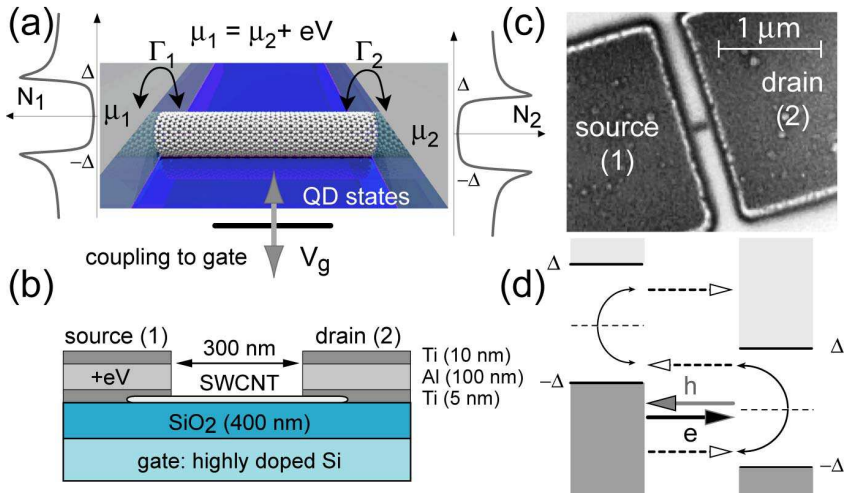

FIG. 1: (a) Illustration of a SWCNT contacted by superconducting (Al) source (1) and drain (2) electrodes. $\mu_{1,2}$ are respectively the electrochemical potentials, $N_{1,2}$ the densityof-states, $\Gamma_{1,2}$ the life-time broadenings due to the coupling of the QD state to the electrodes, $\Delta$ the superconducting gap parameter, $V$ the applied source-drain voltage and $V_{g}$ the gate voltage. (b) Device geometry, showing the evaporated trilayer, consisting of a Ti adhesion layer, the superconducting Al film and a Ti cap-layer. (c) shows an actual device and (d) illustrates possible processes that lead to a subgap current. Shown is in solid a first order Andreev process and dashed a second order one. In the first (second), two (three) quasiparticle (electrons $e$ and holes $h$ ) are involved.

tual superconductor with a bulk critical temperature of $T_{c}=1.2 \mathrm{~K}$. In its thin film form, we rather measure a $T_{c}$ of $0.9 \mathrm{~K}$, which corresponds to a BCS gap-parameter $\Delta_{0}=1.76 k_{B} T_{c}$ of $0.135 \mathrm{meV}$. We drive the Al contacts into the normal state by applying a small perpendicular magnetic field of $B=0.1 \mathrm{~T}$. The substrate is contacted to a third terminal in order to establish a backgate. We measure the differential source(1)-drain(2) conductance $G:=d I / d V$ as a function of source-drain $V$ and gatevoltage $V_{g}$. This is achieved by superposing an ac-voltage $V_{a c}=10 \mu \mathrm{V}$ on $V$ and measuring the corresponding ac current with a current-voltage converter and a lock-in amplifier. Several devices were fabricated and tested at room temperature and at $4.2 \mathrm{~K}$. Here, we focus on a particular interesting device which we selected and measured in a dilution refrigerator. This device has been studied over a large $V_{g}$ window and displays single-electron charging with addition energies in the range of $2 \ldots 5 \mathrm{meV}$. In the following we will focus on a confined gate-voltage regime.

Fig. 2 shows the main measurements we will be focusing on in the following. Visible is in (a) a $d I / d V$ plot in the normal state (n-state) and the corresponding one in the superconducting state (s-state) in (b). In the nstate a sequence of larger and smaller Coulomb blockade (CB) diamonds are seen (dashed lines), corresponding to a sequence of nearly equidistantly spaced levels on the SWNT-QD, which are filled sequentially. The number of electrons on the dot therefore alternates in the groundstate between odd and even [29]. It is also seen that the conductance $G=d I / d V$ around zero bias is suppressed

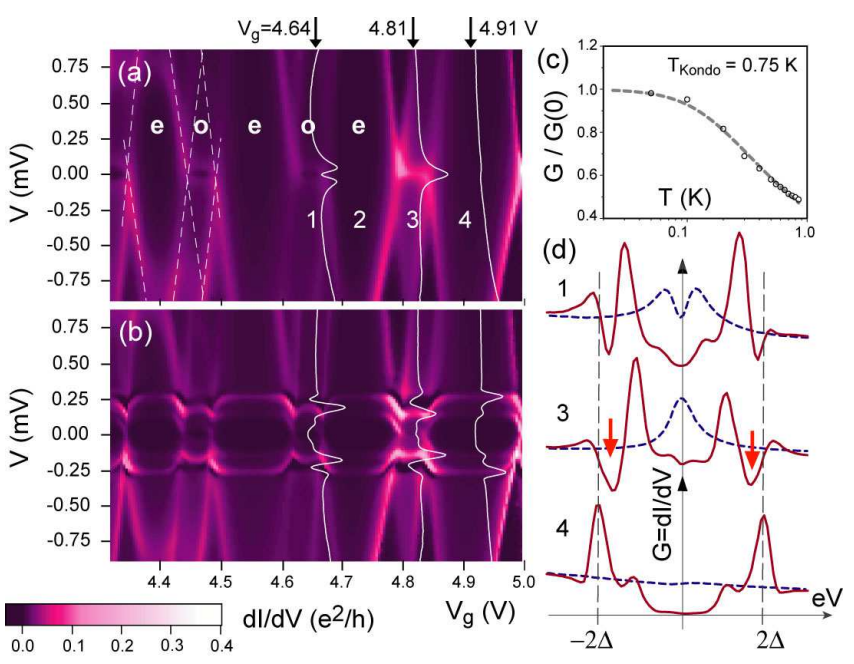

FIG. 2: Differential conductance $d I / d V\left(V, V_{g}\right)$ plot as a function of bias $V$ and gate voltage $V_{g}$ of a SWCNT-QD with superconducting contacts in the normal (a) and superconducting state (b). In (c) we show the linear conductance $G(T)$ measured as a function of temperature $T$ in the middle of charge state 3 . The curves in (d) correspond to the one overlaid on the $d I / d V\left(V, V_{g}\right)$ plots.

and featureless in the even valleys, but is increased assuming structure in the odd ones. In the $\mathrm{CB}$ diamond labelled 3 , there is a pronounced peak at $V=0$, suggesting the appearance of a Kondo resonance. Indeed, the dependence of the linear $G(T)$ on temperature $T$ (Fig. 2c), measured in the middle of this valley, follows the expected dependence [30] with a Kondo temperature of $T_{K}=0.75 \mathrm{~K}$. In the other odd valleys, the Kondo resonances are split by $\approx 0.1 \mathrm{meV}[31$. The origin of this splitting is at present not known, but could be due to exchange with ferromagnetic catalyst particles or another tube (if the present one is a small bundle or contains more than one shell) [32].

The n-state data can be used to deduce a number of parameters. The source, drain and gate capacitances are $C_{1,2} \sim 50,100 \mathrm{aF}$ and $C_{g} \sim 4 \mathrm{aF}$, leading to a gatecoupling $\alpha=C_{g} / C_{\Sigma}$ of $\sim 0.026$, where $C_{\Sigma}=C_{1}+$ $C_{2}+C_{g}$. The charging energy $U=e^{2} / C_{\Sigma}$ and the level spacing $\delta E$ are in the range of $0.7 \ldots 1 \mathrm{meV}$ and $1.4 \ldots 1.8 \mathrm{meV}$, respectively. Whereas this SWNT-QD is nearly symmetric in its electrostatic coupling, it is quite asymmetric in its electronic one. The total level broadening amounts to $\Gamma=\Gamma_{1}+\Gamma_{2} \approx 0.2 \mathrm{meV}$ with an asymmetry of $\Gamma_{1} / \Gamma_{2} \approx 50$. This asymmetry is deduced from the measured current peaks in $d I / d V$ at the border of the $\mathrm{CB}$ diamonds at finite bias and is in agreement with the reduced low temperature zero-bias $G(0)$ of the Kondo ridge 3 , amounting to $G(0) \sim 0.1 e^{2} / h$.

Looking next at the s-state, we see that the major changes in the $d I / d V$ are confined to a voltage band of $-0.26 \mathrm{meV}<V<0.26 \mathrm{meV}$, corresponding to $\pm 2 \Delta$. Above $2 \Delta$, i.e. $|V|>2 \Delta / e$, quasiparticle current is possible and the main modification is caused by the peak 


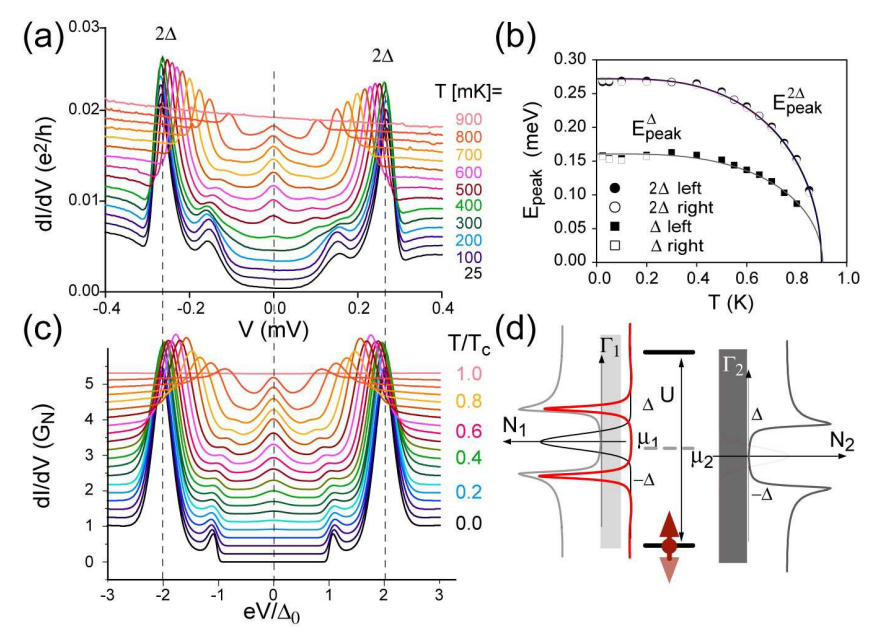

FIG. 3: (a) measured $d I / d V$ versus temperature $T$ in the even valley 4 of Fig. 2a. The $2 \Delta$ and $\Delta$ peak positions $E_{\text {peak }}(T)$ are shown in (b) together with a BCS $T$-dependence of $\Delta$. In (c) we show the result of a model calculation based on tunneling between two superconductors. Good agreement is obtained if the BCS-DOS is broadened by an amount corresponding to $0.2 \Delta_{0}$. The illustration in (d) is our proposal to understand the appearance of the strong $\Delta$ feature in the odd valleys. A Kondo resonance persists on one electrode side only (here, drawn as a thin curve on the left side), pinned to that chemical potential. Due to the gaped DOS, this resonance splits leading to an enhancement of the $\Delta$ feature.

in the superconducting density-of-state (DOS) [3, 11], leading to a peak-like feature in $d I / d V$. Due to the gap in the DOS, first order processes are however suppressed below $2 \Delta$. Depending on the transmission probability [13, 16, 17], higher order Andreev processes can contribute. The first order Andreev process, for example, results in a peak-like structure in the vicinity of $\Delta$. Due to the higher order, the first Andreev peak and all subsequent ones are usually smaller than the quasiparticle one. Both the dominant $2 \Delta$ and the smaller $\Delta$ peak, as well as the suppressed $G$ in the s-state are best visible in the middle of an even charge state (even valley), see e.g. curve labelled 4 in Fig. 2d. In contrast, in the odd charge states, the $2 \Delta$ feature is not present or does not appear at $2 \Delta$. Starting to view the data from large bias voltage, the first peak appears closer to $\Delta$ rather than $2 \Delta$, with a preceding negative $d I / d V$ (NDR), see curve labelled 3 in Fig. 2d. Hence, there is a striking even-odd asymmetry in the finite-bias $d I / d V$ features in the s-state which is not caused by the $\mathrm{CB}$ resonance at the edges of adjacent charge states, where the situation is expected to be more complex [16, 17]. The even-odd alteration of the MAR structure suggest a relation to Kondo physics. To model this, we first extract important parameters from an analysis of the data in the middle of an even valley where Kondo correlations are absent.

Fig. 3a-c discusses the temperature dependence of $d I / d V$ in the middle of the even charge state; (a) shows the measurement taken in valley 4 of Fig. 2 and (c) is a model calculation. The experiment displays pronounced quasiparticle current peaks at $E_{\text {peak }}= \pm 2 \Delta$, and weaker MAR peaks at $\pm \Delta$. The evolution of $E_{\text {peak }}^{j \Delta}(T)$ with temperature $T$ are shown in Fig. 3b together with an approximate BCS gap function $E_{\text {peak }}^{j \Delta}=$ $K_{j \Delta} \Delta_{0} \tanh \left(1.74 \sqrt{T_{c} / T-1}\right)$, where we used the BCS value for $\Delta_{0}=1.76 k_{B} T_{c}$, which amounts to $0.135 \mathrm{meV}$ for a $T_{c}$ of $0.9 \mathrm{~K}$. We then obtain $K_{2 \Delta}=2.0$ and $K_{\Delta}=1.15$ for the two peaks. The slight increase of $K_{\Delta}$ above the expected value of 1 is not unusual. We will be using the value $\Delta_{0}=0.13 \mathrm{meV}$ as the zero- $\mathrm{T}$ gap parameter in the following. The relevant parameters expressed in units of $\Delta_{0}$ are then: $U=5 \ldots 8, \delta E=10 \ldots 14$, $\Gamma \approx 1.5$ and $T_{K} \approx 0.5$. The zero-bias peak in Fig. 3a, appearing at intermediate temperatures, can be explained by direct tunneling of quasiparticles thermally activated across the gap.

The good agreement with the BCS relation of the peakpositions motivates the modelling of the $d I / d V$ using the BCS-DOS in the leads. Although we would have to use a theory describing resonant tunneling between two superconductors, such as the one from Levy Yayati et al. [16], a simple tunneling picture suffices [33], because the resonant levels are far away from the electrochemical potential of the electrodes in the middle of a charge state. The subgap current is treated in the same approximation using [11]. To obtain a reasonable fit, the BCS-DOS has been convoluted with a Gaussian of width $\eta$. A quite good agreeement is found with $\eta=0.2 \Delta_{0}$.

We now turn our attention to the odd charge states. We point out, that the zero-bias high- $G$ Kondo 'ridge', which is associated with the Kondo resonance and visible in the n-state, is not seen in the s-state. This is consistent with a Kondo temperature $T_{K}$ that is smaller than $\Delta$, i.e. $T_{K}=0.5 \cdot \Delta[2,12]$. Although the Kondo resonance is not visible in the s-state, we suspect it to be responsible for the even-odd asymmetry of the $\Delta$-feature in the sstate.

In the Kondo regime, the single spin on the QD in the odd state is screened by exchange with conductance electrons from the leads. If the quasiparticles are bound in Cooper pairs in the leads, the Kondo temperature is renormalized, assuming a smaller value $T_{K}^{\star}$. This renormalization is sensitive on the actual parameters $\Gamma_{1,2}$ and $\Delta$. Due to the asymmetry, it may happen that a Kondo resonance with a reduced width forms on the contact with the larger $\Gamma$, whereas on the other one it is suppressed. This is illustrated in Fig. 3d.

We modelled this scenario considering a single-level Anderson Hamiltonian with interaction $U=5 \ldots 10 \Delta_{0}$ coupled to source and drain contacts. The experimentally deduced $\Gamma$ 's, including the strong asymmetry were used. The calculation is based on a slave-boson meanfield treatment of this interacting problem [21]. The result of the comparison is shown in Fig. 4: (a) corresponds to the n-state and (b) and (c) to the s-state. Despite this simple model, the agreement is surprisingly good. It is 

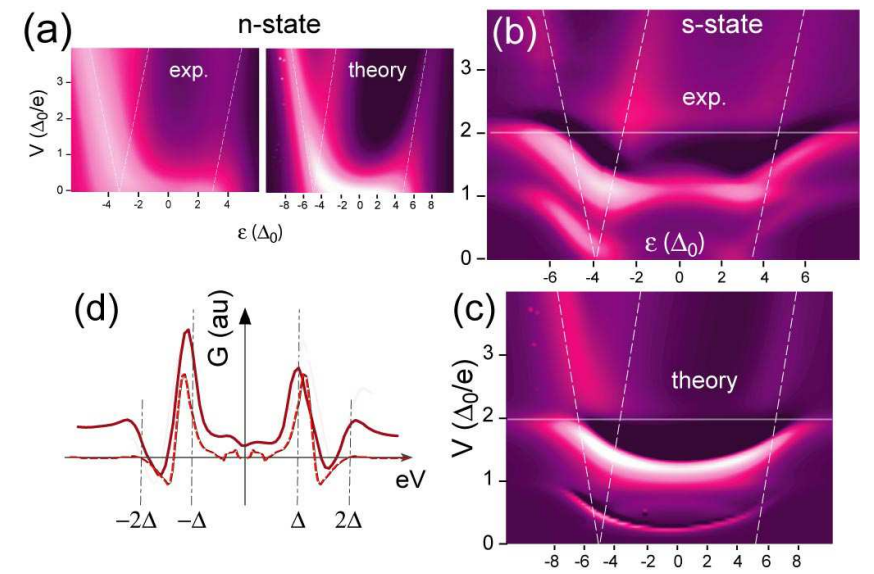

FIG. 4: Comparison of the $d I / d V$ in the odd valley labelled 3 in Fig. 2a with a model caluclation based a single-level Anderson model with interaction $U$, that is evaluated by a meanfield slave-boson ansatz. The n-state is shown in (a), the s-state in (b) and (c). $\epsilon$ denotes the level position. The experiment corresponds to $U=7-8$ (in units of $\Delta_{0}$ ), whereas the calulation was done for $U=5$ and $U=10$, where the latter is shown here. The upper solid (dashed) curve in (d) is cross-sections in the s-state at $\epsilon=0$, taken from the experiment (theory).

remarkably good in the normal state, shown in Fig. 4a. In the s-state, the dominance of the $\Delta$-like feature in the odd valley is clearly present, as is a similar cross-over from odd to even filling. There are also some differences: in the experiment the $\Delta$-feature bends to larger $V$-values in the middle of the odd state, whereas this feature is rather flat in the calculation.

In conclusion we have discovered a pronounce evenodd effect in the (multiple) Andreev structure in transport through a QD with superconducting contact. This effect is driven by a Kondo resonance pinned to one contact only and defines a new regime. Whereas a high conductance channel from source to drain, driven by Kondo correlations, persists in the supercondcuting state if $T_{K} \gg \Delta$, this channel is greatly suppressed in the opposite limit. In the intermediate regime $T_{K} \sim \Delta$, and in particular for asymmetric dot-electrode couplings, the (partial) Kondo-screening of the 'impurity' spin may occur on one electrode only. It would be interesting to explore the 'robustness' of this feature in model calculation and to fabricate similar QDs with tunable electrode couplings.

\section{ACKNOWLEDGEMENTS}

We have profited from fruitful discussions with E. Scheer, W. Belzig, and V. Golovach. We thank J. Gobrecht (PSI) for providing the oxidized Si substrates. The work at Basel has been supported by by the Swiss National Science Foundation, the NCCR on Nanoscale Science, FP6-RTN DIENOW and EU-FP6-IST project HYSWITCH.
[1] For a recent review, see: Mahn-Soo Choi, Int. J. of Nanotechn. 3, 216 (2006).

[2] L. I. Glazman and K. A. Matveev, JETP Lett. 49, 659 (1989).

[3] D. C. Ralph, C. T. Black, and M. Tinkham, Phys. Rev. Lett. 743241 (1995);

[4] A. F. Morpurgo, J. Kong, C. M. Marcus, and H. Dai, Science 286, 263 (1999).

[5] for a review, see: N. Agrait, A. Levy Yeyati, J. M. van Ruitenbeek, Phys. Rep. 377, 81 (2003).

[6] A. Yu. Kasumov, R. Deblock, M. Kociak, B. Reulet, H. Bouchiat, I. I. Khodos, Yu. B. Gorbatov, V. T. Volkov, C. Journet, and M. Burghard, Science 284, 1508 (1999);

[7] P. G. de Gennes, Rev. Mod. Phys. 36, 225 (1964); A. F. Andreev, Sov. Phys. JETP 19, 1228 (1964);

[8] B. D. Josephson Rev. Mod. Phys. 36, 216 (1964); K. K. Likharev, Rev. Mod. Phys. 51, 101 (1979).

[9] M. Octavio, M. Tinkham, G. E. Blonder, and T. M. Kalpwijk, Phys. Rev. B 27, 6739 (1983).

[10] For a review, see: special issue on Mesoscopic Superconductivity, Superlattices and Microstructures 25, issues 5/6, edited by P. F. Bagwell (1999).

[11] J. C. Cuevas, A. Martín-Rodero, and A. Levy Yeyati Phys. Rev. B 54, 7366 (1996).

[12] M. R. Buitelaar, T. Nussbaumer, C. Schönenberger, Phys. Rev. Lett. 89, 256801 (2002).
[13] M. R. Buitelaar, W. Belzig, T. Nussbaumer, B. Babic, C. Bruder, and C. Schönenberger, Phys. Rev. Lett. 91, 057005 (2003).

[14] M. R. Gräber, T. Nussbaumer, W. Belzig, and C. Schönenberger, Nanotechnology 15, S479-82 (2004).

[15] P. Jarillo-Herrero, J. A. van Dam, and L. P. Kouwenhoven, Nature 439, 953 (2006); H. I. Jorgensen, K. Grove-Rasmussen, T. Novotný, K. Flensberg, and P. E. Lindelof, Phys. Rev. Lett. 96, 207003 (2006); J.-P. Cleuziou, W. Wernsdorfer, V. Bouchiat, T. Ondarcuhu, M. Monthioux, Nature Nanotechnol. 1, 53 (2006).

[16] A. Levy Yeyati, J. C. Cuevas, A. López-Dávalos, and A. Martín-Rodero, Phys. Rev. B 55, R6137 (1997).

[17] G. Johansson, E. N. Bratus, B. Verkin, V. S. Shumeiko, and G. Wendin, Phys. Rev. 60, 1382 (1999).

[18] D. Goldhaber-Gordon, H. Shtrikman, D. Mahalu, D. Abush-Magder, U. Meirav, and M. A. Kastner, Nature 391, 156 (1998).

[19] M. Pustilnik, L. Glazman, J. of Phys.: Cond. Matter 16, R513 (2004).

[20] P. Schwab and R. Raimondi, Phys. Bev. B 59, 1637 (1999); S. Y. Liu and X. L. Lei, Phys. Rev. B 70, 205339 (2004).

[21] F. S. Bergeret, A. Levy Yeyati, and A. Martín-Rodero, Phys. Rev. B 74, 132505 (2006).

[22] J. C. Cuevas, A. Levy Yeyati, and A. Martín-Rodero, 
Phys. Rev. B 63, 094515 (2001).

[23] A. Levy Yeyati, A. Martín-Rodero, and E. Vecino, Phys. Rev. Lett. 91, 266802 (2003); Y. Avishai, A. Golub, and A. D. Zaikin, Phys. Rev. B 67, 041301 (2003).

[24] S. Sapmaz, P. Jarillo-Herrero, L. P. Kouwenhoven, H. S. J. van der Zant, Semicond. Sci. Technol. 21, S52 (2006).

[25] J. Nygard, D, H. Cobden, P. E. Lindelof, Nature 408, $342(2000)$

[26] Y.-J. Doh, J. A. van Dam, A. L. Roest, E. P. A. M. Bakkers, L. P. Kouwenhoven, and S. De Franceschi, Science 309, 272 (2005); J. A. van Dam, Y. V. Nazarov, E. P. A. M. Bakkers, S. De Franceschi, and L. P. Kouwenhoven, Nature 442, 667 (2006).

[27] J. Kong, H. T Soh, A. M. Cassel, C. F. Quate, and H. Dai, Nature 395, 878 (1998).

[28] at $T=950^{\circ}$ in a mixture of $\mathrm{H}_{2}$ and $\mathrm{CH}_{4}$ with flow rates of 0.5 and $1.0 \mathrm{l} / \mathrm{min}$, respectively.

[29] D. H. Cobden, M. Bockrath, P. L. McEuen, A. G. Rin- zler, R. E. Smalley, Phys. Rev. Lett. 81, 681 (1998); D. H. Cobden, and J. Nygard, Phys. Rev. Lett. 89, 046803 (2002).

[30] D. Goldhaber-Gordon et al., Phys. Rev. Lett. 81, 5225 (1998).

[31] This splitting of the Kondo resonace is much larger than the Zeeman splitting due to the applied magnetic field of $B=0.1 \mathrm{~T}$, amounting to $<10 \mu \mathrm{eV}$.

[32] H. Jeong, A. M. Chang, and M. R. Melloch, Science 293, 2221 (2001); A. N. Pasupathy, R. C. Bialczak, J. Martinek, J. E. Grose, L. A. K. Donev, P. L. McEuen, and D. C. Ralph, Science 306, 86 (2004); J. Nygard, W. F. Kohl, N. Mason, L. DiCarlo, and C. M. Marcus, cond-mat/0410467. M. G. Vavilov and L. I. Glazman, Phys. Rev. Lett. 94, 086805 (2005).

[33] M. Tinkham, Introduction to Superconcuctivity (MacGraw Hill, New York 1996). 